\title{
Tratamiento Grupal Intensivo del Tabaquismo
}

\author{
SERGIO BELLO S.* y HAYDÉE CHAMORRO R.**
}

\section{Intensive Smoking Cessation Group Therapy}

We describe the methodology used at the Instituto Nacional del Tórax for smoking cessation, group therapy. We carried out 7 weekly sessions, where the principles of the Motivational Interview are used. There is a first stage of preparation (3 sessions), fixed D-day and then 4 sessions of follow up, for the management of deprivation syndrome, relapse prevention and maintenance of abstinence.

Key words: Smoking cessation; Psychotherapy group; motivational interviewing; counseling.

\section{Resumen}

Se describe la metodología utilizada en el Instituto Nacional del Tórax para el tratamiento del tabaquismo, modalidad grupal. Se realizan 7 sesiones semanales, donde se utilizan los principios de la Entrevista Motivacional. Hay una primera etapa de preparación (3 sesiones), se fija día D y luego 4 sesiones de acompañamiento, manejo del síndrome de privación, prevención de recaídas y mantención de la abstinencia.

Palabras clave: Dejar de fumar; psicoterapia de grupo; entrevista motivacional; consejería.

En el tratamiento intensivo del tabaquismo se utilizan las mismas herramientas que en cualquier consejería en tabaco ${ }^{1}$, incluida la breve, sólo que con más tiempo de dedicación. Estas herramientas son la entrevista motivacional, las etapas de cambio y las $5 \mathrm{~A}$ y $5 \mathrm{R}$.

Se describe a continuación la metodología utilizada en el Instituto Nacional del Tórax ${ }^{2}$ en su modalidad grupal, que se privilegia por su costo-efectividad.

El abordaje psicosocial del tabaquismo se realiza a través de estrategias cognitivo-conductuales, además de apoyo farmacológico con alguna de las tres drogas consideradas de primera línea (terapia de reemplazo de nicotina, bupropión y vareniclina). La terapia grupal está estructurada de manera tal que permita a los pacientes un trabajo personalizado inserto en un contexto de grupo, considerando las dimensiones educacional, psicológica y social.

El objetivo es lograr que los participantes del grupo de tratamiento abandonen el consumo de tabaco a través del desarrollo de estrategias y habilidades para ello. Se trabaja en el reconocimiento de la adicción y la modificación de pautas de comportamiento, orientadas a cambiar estilos de vida y modificar la identidad de fumador a no-fumador.

Todos los pacientes fumadores son evaluados en forma individual a través de una entrevista clínica en que se evalúa la adicción a través del test de Fagerström, la motivación para dejar de fumar a través del test de Richmond, el estado psico-emocional con el test de Goldberg y la etapa del proceso de cambio de Prochaska en que se encuentran. El único criterio de exclusión es tener 5 o más puntos en el Test de Goldberg de 12 preguntas, que hace probable un trastorno depresivo/ansioso. Dichos pacientes son derivados a salud mental y se les deja abierta la posibilidad de volver una vez estabilizados por tres meses.

* Profesor Adjunto, Facultad de Medicina e INTA. Universidad de Chile. Programa de Tabaquismo. Instituto Nacional del Tórax.

** Experta en Tabaquismo. Programa de Tabaquismo. Instituto Nacional del Tórax. 


\section{Tratamiento Grupal}

Después de la evaluación inicial se integran a terapia grupal, cada uno de ellos conformado por 8-15 pacientes. La terapia grupal consta de 7 sesiones, con una duración de 1,5 a $2 \mathrm{~h}$, una vez a la semana, en que participan dos profesionales capacitados en terapia del tabaquismo y manejo de grupos. Se realiza control telefónico intra tratamiento (entre una sesión y otra) y con posterioridad al término se hace una sesión presencial 1 mes después, además de seguimiento telefónico, utilizando protocolos pre- establecidos.

\section{Descripción del proceso terapéutico durante la terapia grupal}

Se utilizan los principios de la Entrevista Motivacional ${ }^{3,4,5}$ buscando, a través de la empatía y el desarrollo de las discrepancias, evocar en cada fumador la mejor motivación para el cambio. Los terapeutas entregan a los fumadores el protagonismo en el proceso de lograr y mantener la abstinencia. Se establece así, una relación horizontal terapeutas - grupo, creándose un clima caracterizado por calidez, aceptación incondicional, apertura y valoración de la experiencia de los otros. En este contexto, cada participante es a la vez paciente y terapeuta del grupo, ya que cada vivencia compartida en el grupo adquiere un valor formativo.

Las 3 primeras sesiones corresponden al período de preparación para dejar de fumar. Se enfocan en construir un clima de confianza básica, conocer las características de la adicción, mostrar los daños producidos por el consumo de tabaco y aumentar la motivación para abandonar el tabaquismo. Se identifican las condicionantes de la conducta fumadora y se entregan herramientas para su manejo.

Entre las sesiones 3 y 4 se fija el "día D" (primer día sin fumar), donde la terapia se enfoca en el manejo del síndrome de privación, la prevención de recaídas y mantención de la abstinencia. El logro de estos objetivos se basa en técnicas de fortalecimiento de la autoconfianza, autoeficacia, refuerzo individual y social, técnicas de manejo de tensiones (ansiedad y craving) y con apoyo farmacológico iniciado en la semana previa. Es relevante trabajar las vivencias que surgen al poner fecha al primer día sin fumar.

Las sesiones 5 y 6 se centran en valorar los beneficios asociados al dejar de fumar y a transformar un compromiso formal en cambio conductual duradero previniendo recaídas. Para lograr este objetivo, sesión a sesión se les van entregando diversas herramientas: técnicas de relajación, estrategias para enfrentar las tentaciones, entrenamiento en asertividad, refuerzo individual y social. Las técnicas empleadas permiten a los participantes ponerse en contacto con ellos mismos, explorar ganancias secundarias al remitir la conducta problema, identificar y controlar conductas automáticas, reforzar la decisión de abstinencia, logrando no sólo la meta prevista, sino además objetivos secundarios de fortalecimiento de autoestima y autoeficacia, que instan a tomar otras decisiones en pro de enriquecer y mejorar sus estilos de vida. Por otra parte, la potencia del refuerzo social al interior del grupo queda de manifiesto en las últimas sesiones, al fomentar la motivación de aquellos fumadores que evidencian mayor dificultad para lograr la abstinencia.

En la sesión 7 se consolida el apoyo grupal, se evalúa el tratamiento y se proyecta la abstinencia en el tiempo.

Se realiza seguimiento telefónico y una sesión presencial, un mes después del término del periodo semanal, para consolidar la abstinencia y revisar el o los fármacos utilizados.

\section{Bibliografía}

1.- SALINAS J, BELLO S, CHAMORRO H, GONZÁLEZ CG. Consejería en alimentación, actividad física y tabaco. Instrumento fundamental en la práctica profesional. Rev Chil Nutr 2016; 43,:434-42.

2.- BELLO S, CHAMORRO H, ORELLANA M, CISTERNAS L, SALINAS M. Experiencia de un programa de cesación del tabaquismo en el Instituto Nacional del Tórax. Rev Chil Enferm Respir 2015; 31: 94-100.

3.- BALCELLS M, TORRES M, YAHNE C. La terapia motivacional en el tratamiento del tabaquismo. Adicciones 2004; 16 (supl 2): 227-36.

4.- LIZARRAGA S, AYARRA M. Entrevista Motivacional. Revista ANALES Sis San Navarra 2001; 24 (Suplemento 2): 43-53.

5.- LÜHNING S, BULJUBASICH D. Entrevista motivacional: la nueva estrella de la película. Prev Tab 2014; 16: 7-11.

Correspondencia a:

Dr. Sergio Bello S.

Email: sbello@torax.cl 